\title{
Design and Optimization of an EBG Antenna with an Efficient Electromagnetic Solver
}

\author{
Josefa Gómez, Abdelhamid Tayebi, José Ramón Almagro, Iván González, and Felipe Cátedra \\ Computer Sciences Department, University of Alcalá, 28871 Alcalá de Henares, Spain \\ Correspondence should be addressed to Josefa Gómez, josefa.gomezp@uah.es \\ Received 28 June 2011; Revised 3 September 2011; Accepted 15 September 2011 \\ Academic Editor: Ning Yuan \\ Copyright () 2012 Josefa Gómez et al. This is an open access article distributed under the Creative Commons Attribution License, \\ which permits unrestricted use, distribution, and reproduction in any medium, provided the original work is properly cited. \\ A novel electromagnetic bandgap (EBG) antenna has been designed, optimized, and analyzed using an efficient electromagnetic \\ solver based on the moment method. Very good agreement between simulations and measurements in anechoic chamber has been \\ obtained. Comparisons of the antenna with and without the EBG structure have been conducted to study its influence in terms of \\ gain. This new EBG antenna is an excellent candidate for several applications due to its high gain and good polarization purity.
}

\section{Introduction}

Recently, several applications of EBG materials, such as microwave filters, antennas, amplifiers, microstrip devices, ground plane structures, and base station antennas have been reported in the literature. For instance, EBG material has been shown to enhance the directivity of a patch antenna from $8 \mathrm{~dB}$ to $20 \mathrm{~dB}$ [1]. The work presented in [2] described a method to design directive antennas by incorporating cylindrical EBG structures. On the other hand, [3] presents a resonator antenna that increases its gain and bandwidth with an EBG structure over the device. It has also been demonstrated [4] that the EBG materials are able to provide good results while reducing the size of some kinds of filters. The work presented in [5] summarizes the benefits of using EBG materials to improve the performance of microwave and optical applications. Some experimental results are discussed to check the properties of these materials. According to [6], an increase in directivity can be obtained by adding a partially reflecting sheet in front of the antenna because of the multiple reflections between the sheet and the screen. The resonance distance between both elements must be such that the rays projected through the sheet have equal phases in the normal direction. Our contribution is focused on that investigation line. Numerical results demonstrate an improvement in gain, bandwidth, and polarization purity when comparing several antenna configurations varying the distances between both grids. The aim of this paper is to design a compact EBG antenna that provides a gain greater than $15.0 \mathrm{dBi}$ and polarization purity greater than $40 \mathrm{~dB}$ while maintaining a very small size. To fulfill these requirements, the dimensions of the antenna were optimized by using a powerful electromagnetic solver.

The paper is organized as follows. Section 2 presents the main characteristics of the electromagnetic field solver that has been used to design, optimize, and analyse the proposed antenna. The steps of the optimization process that have been conducted to find the optimum dimensions of the antenna are listed in Section 3. Section 4 describes the properties of the EBG antenna as well as the design and analysis procedure. Section 5 includes the comparisons between predictions and measurements and Section 6 contains the concluding remarks and future work.

\section{Electromagnetic Kernel}

In recent years, interest in computer simulation tools has grown rapidly. Many people in academia and industry are currently involved in the research and development of new methods to accelerate or improve the conventional electromagnetic field solvers. The applications of this kind of systems are unlimited (design of microwave devices, analysis of antennas, radomes, reflectarrays, electromagnetic compatibility, etc.) and they can be used not only in engineering, but also in other scopes like medicine [7]. On the other hand, researchers may take advantage of the parallel versions due 


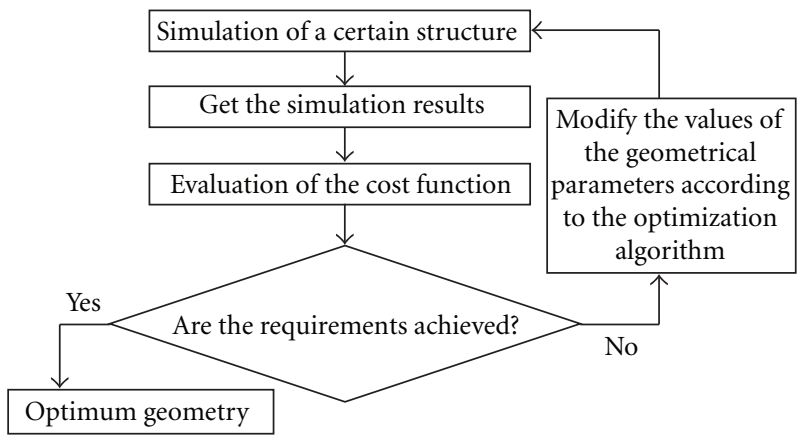

Figure 1: Diagram of the optimization process.

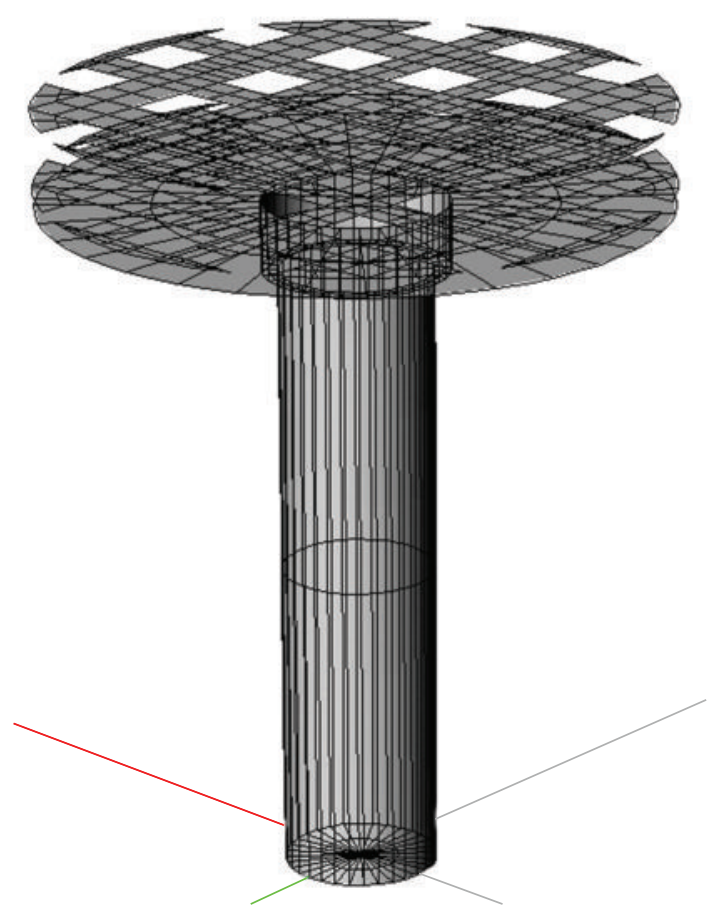

(a)

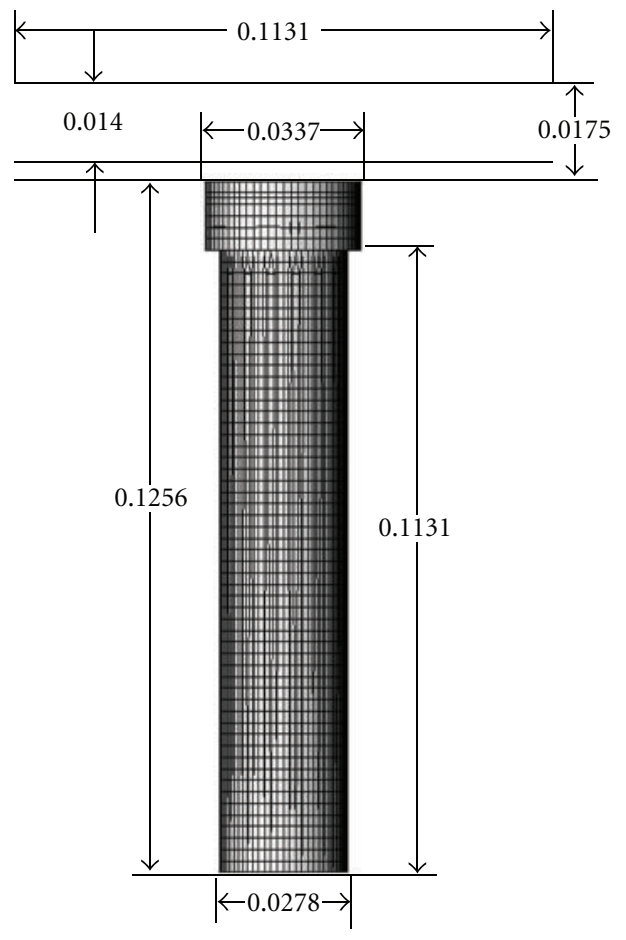

(b)

FIGURE 2: Schematic representation of the antenna. Units are in meters.

to the new power characteristics of actual computers such as high speed and enormous amount of memory.

Nowadays, many commercial suites similar to the computer tool utilized to perform the simulations like Feko, CST, and HFSS are available. However, none of them takes into account the real shape of the bodies under analysis. This is because the meshing procedure is based on flat facets (triangles or quadrangles), so that small geometrical details can be lost. One of the advantages of our solver is that the discretized geometry totally fits the original shape of the structure, because it is composed of curved parametric surfaces. As a result, it provides accurate predictions of arbitrary metallic or dielectric 3D structures at low and high frequencies [8].

As previously mentioned, the electromagnetic kernel is based on the moment method [9] and can solve the electrical field integral equation, the magnetic field integral equation and the combined field integral equation. The electromagnetic treatment considers curved rooftop functions that serve as low-level basis and testing functions. Both functions are defined over a parametric domain, which is very well suited for setting up an adaptive mesh, without the need for external meshing tools. Other important advantage of using these functions is that their direct placement on parametric surfaces totally eliminates the error introduced when using plane facets in the discretization process.

To verify the efficiency of the tool, some comparisons with Feko have been conducted. The conclusion obtained is that Feko is usually three times slower than our electromagnetic solver. A pyramidal horn analysis problem at $10 \mathrm{GHz}$ can be used as a benchmark example to demonstrate the efficiency of the solver. Whilst the simulation in Feko spends 


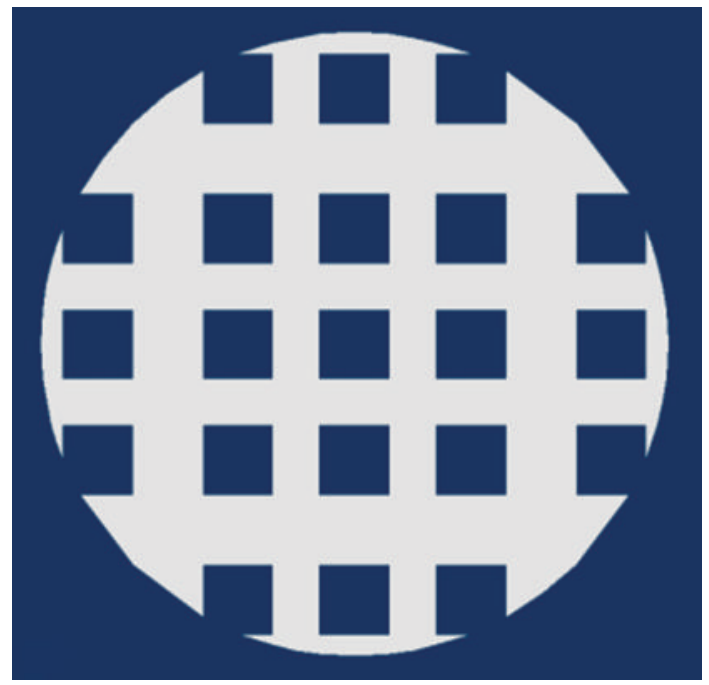

(a)

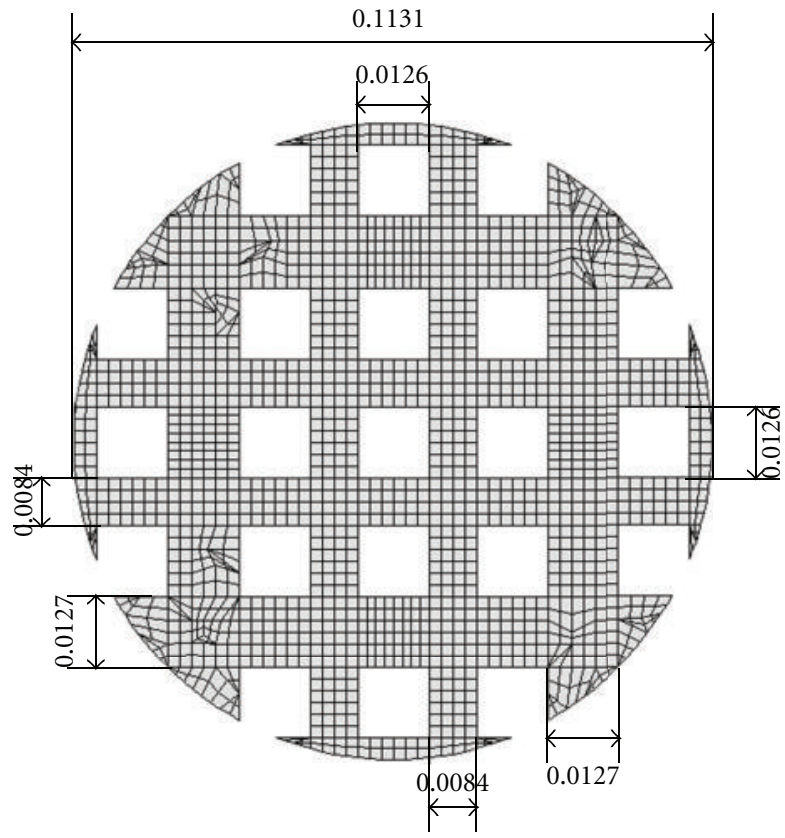

(b)

Figure 3: Top view of the EBG structure. Units in meters.

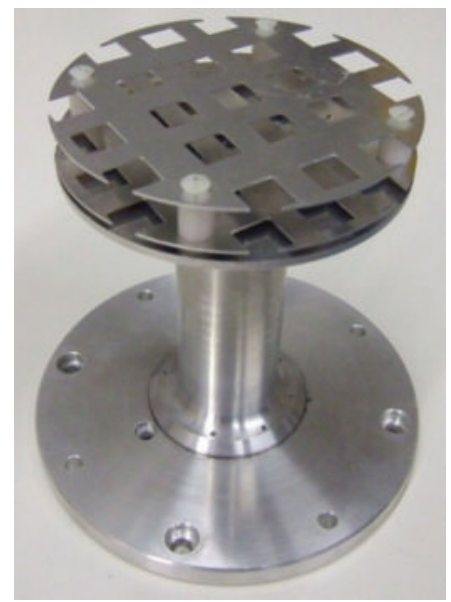

Figure 4: Prototype of the EBG antenna.

183 seconds solving the matrix with LU decomposition and 7378 unknowns, our computer tool only spends 84 seconds solving the same problem with an iterative method and 2205 unknowns.

Due to the fact that conventional moment method spends lots of resources when the structure under analysis is electrically large, the multilevel fast multipole algorithm (MLFMA) [10] has been included in the electromagnetic kernel to improve the efficiency in those cases. MLFMA only requires the storage of the near-field terms of the coupling matrix, so that it decreases the CPU-time of the matrix-vector products in the solution process, allowing the analysis of problems of sizes of thousands of wavelengths.

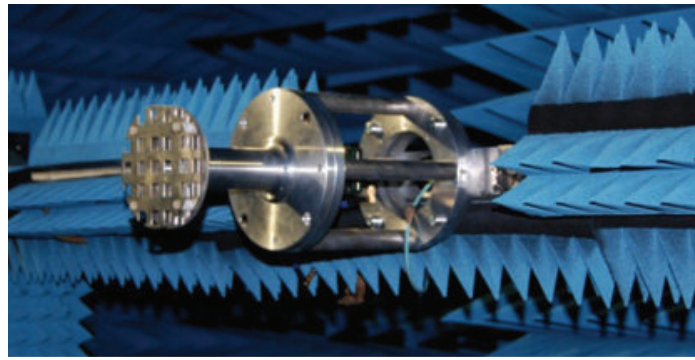

FIGURE 5: Antenna located in the anechoic chamber.

The computational complexity is therefore reduced from $\mathrm{O}\left(\mathrm{N}^{2}\right)$ to $\mathrm{O}(\mathrm{N} \log \mathrm{N})$. The benefit of applying this method is a dramatic reduction of memory and time resources needed for simulation of electrically large structures.

The kernel also includes a hybrid computational technique based on the moment method and physic optics [11] for analysis of large antennas and scatters at high frequencies. A combination of asymptotic and rigorous techniques is the ideal solution when a large body is too hard to analyze with the MLFMA. The inclusion of physic optics helps to reduce the computation time and memory requirements with respect to the conventional moment methods when analysing huge bodies at high frequencies.

On the other hand, a domain decomposition approach [12] has been incorporated to obtain similar results to those given by the moment method, highly reducing the computational effort by taking advantage of the MLFMA method combined with an iterative technique used to compute interactions between different parts of the geometry in a totally adaptive fashion. The domain decomposition 
Radiation pattern, $f=9 \mathrm{GHz}, \phi=0$

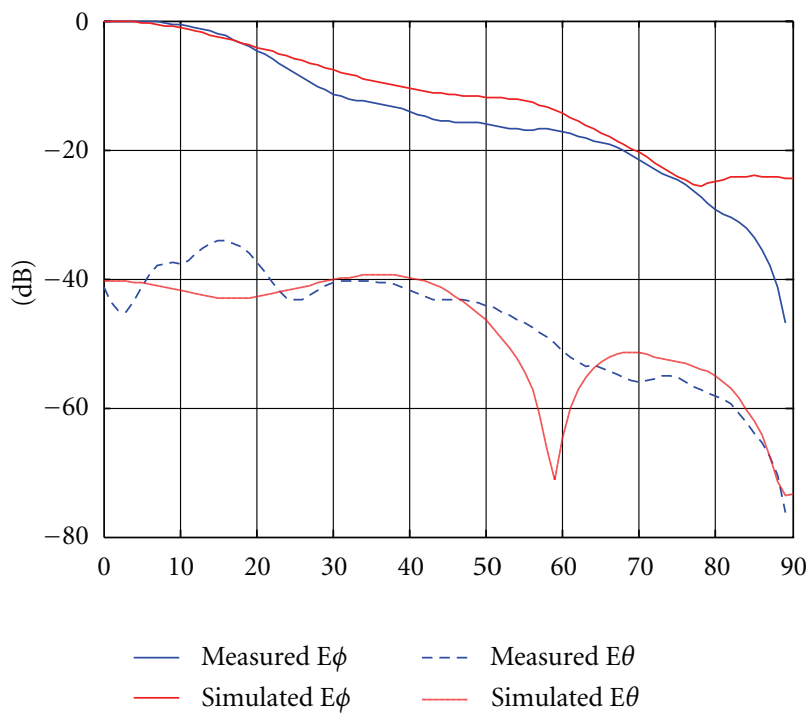

Figure 6: Comparison of the simulated and measured radiation patterns. E-plane cut at $9 \mathrm{GHz}$.

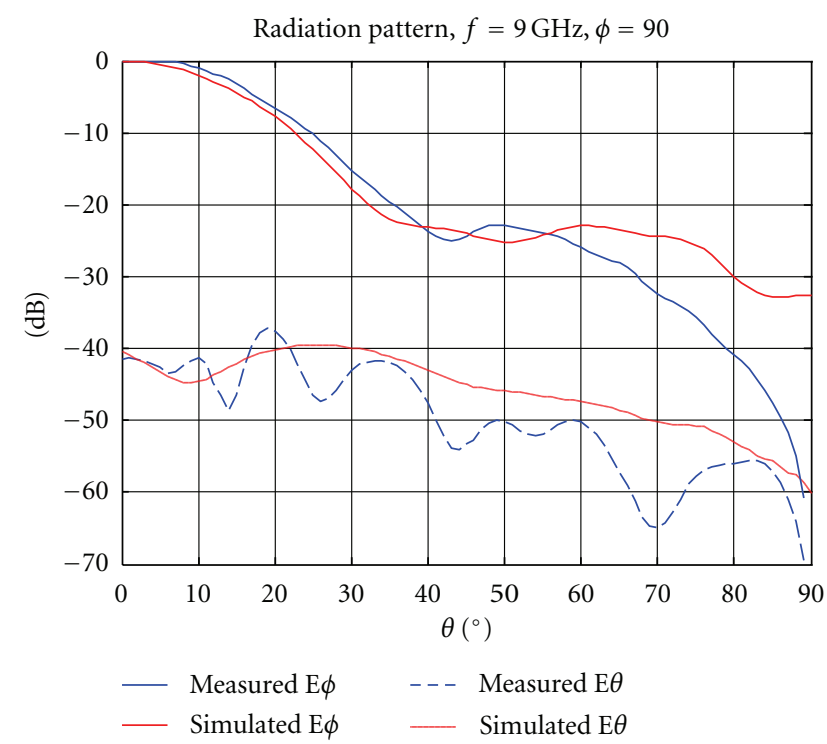

FIgURE 7: Comparison of the simulated and measured radiation patterns. H-plane cut at $9 \mathrm{GHz}$.

approach entails a simple way to deal with a good number of realistic problems which due to convergence or size limitations cannot be handled by conventional rigorous solvers, but are not included in the field of application of highfrequency techniques either. These geometries can include structures containing fine details, multiscale problems, interactions with cavities, and so forth.

Lately, the use of computer clusters to perform electromagnetic analysis of many realistic problems in researching centers is quite common. For this purpose, the kernel has been parallelized using the message passing interface paradigm [13], a specification for an API used to program parallel computers. The goals of this paradigm are high

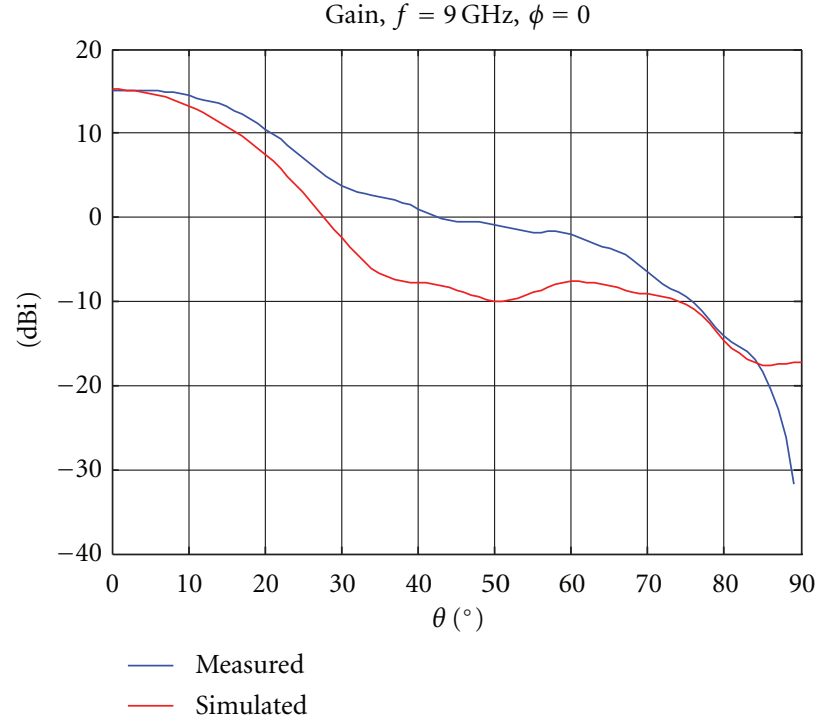

FIGURE 8: Comparison of the simulated and measured gain. E-plane cut at $9 \mathrm{GHz}$.

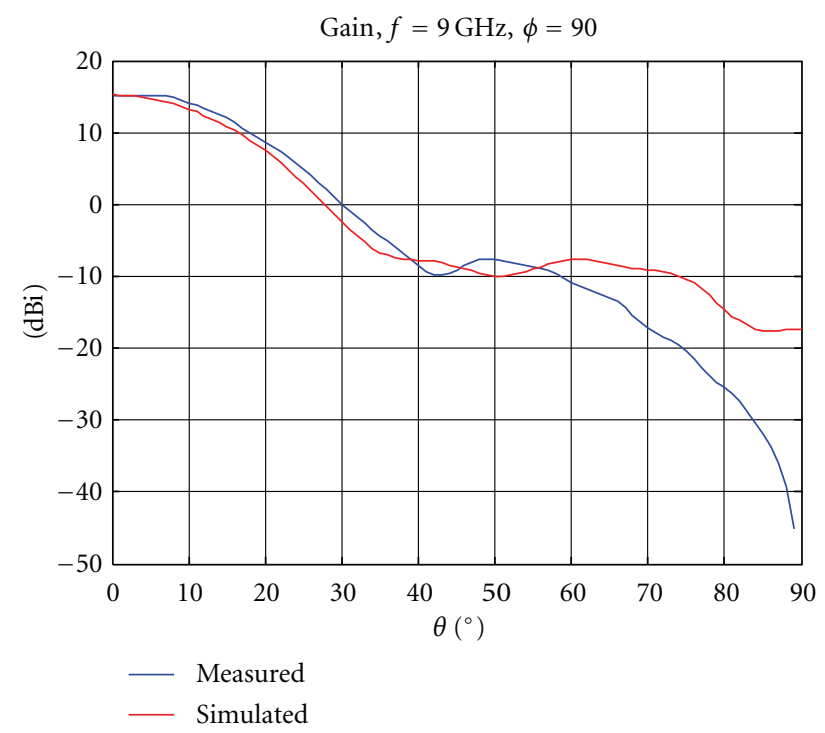

FIGURE 9: Comparison of the simulated and measured gain. Hplane cut at $9 \mathrm{GHz}$.

performance, scalability, and portability. The computer tool is compatible with any modern Windows PC or Linux-based machine and there are also versions available for other operating systems and platforms, including highly parallelized versions for multiprocessor computers or clusters.

\section{Optimization Process}

One of the most important features of the solver is the optimization process $[14,15]$. This module computes the optimal geometric values of a number of parameters previously indicated by the user (width, radius, height, antenna 

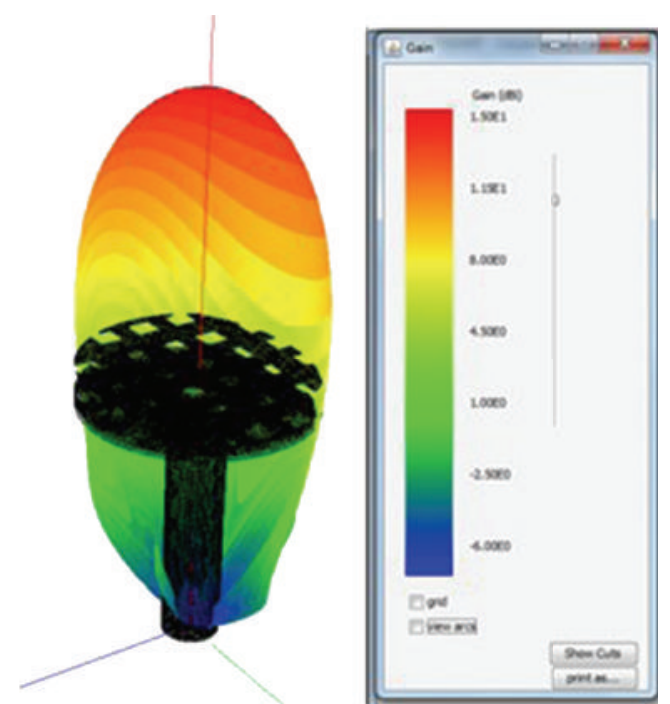

Figure 10: Radiation pattern of the antenna computed by the electromagnetic simulator.

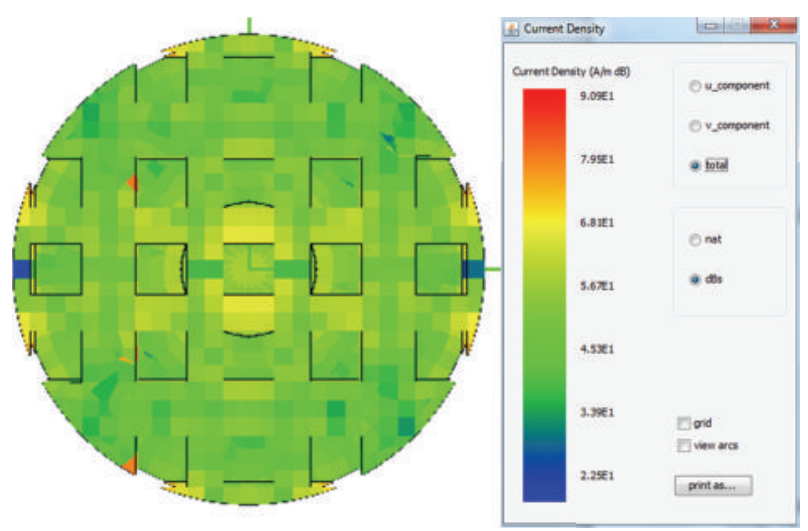

Figure 11: Current distribution computed by the electromagnetic simulator (top view).

location, etc.). The process allows the optimization of radiation patterns and it is based on several specifications set out initially by means of a cost function. Thus, the shape of the radiation pattern can be defined in terms of gain, level of side lobes, level of the crosspolar component, and so forth. The selected parameters vary within a known range, in order to find the optimal solution that minimizes this cost function.

The process begins by analyzing a particular structure, defined by initial parameters. At the end of the simulation, results are processed and the cost function is evaluated. If all requirements are satisfied, the values of the parameters that have provided such results are given as the optimum parameters. If any specification is not satisfied, the geometry is updated with new parameters and a new simulation is performed, repeating the whole process. The optimization procedure finishes when the optimizer minimizes the specified cost function. Figure 1 depicts a diagram of the whole process.

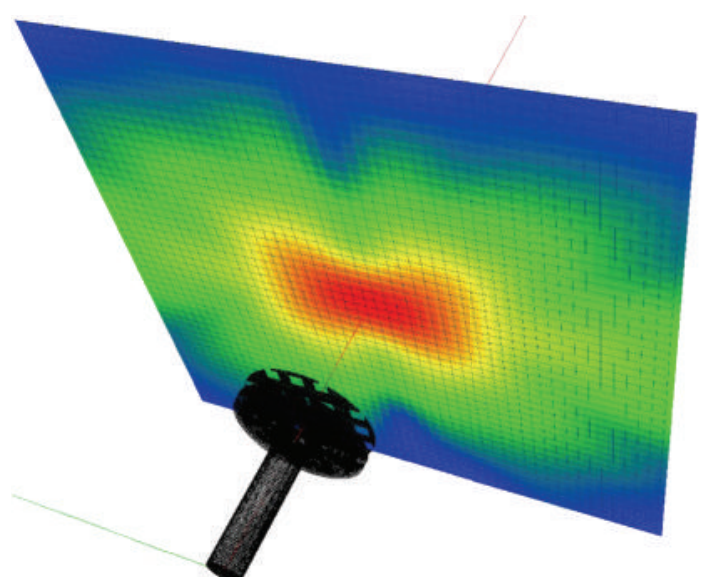

FIGURE 12: Near field results computed by the tool considering the $50 \times 50$ observation points.

Regarding the optimization algorithm, there exist three different methods that can be selected. The first one is based on an exhaustive search, since each parameter is updated with values taken from a homogeneous grid. gradient descend and particle swarm optimization are two popular algorithms that have been also included in the optimization module. Successful results have been achieved in the optimization of several antenna dimensions when applying these algorithms.

\section{EBG Antenna}

In order to design a compact EBG antenna that provides high gain, polarization purity, and wide bandwidth, the dimensions were optimized by using the optimization module. After obtaining the optimum dimensions, a prototype made of aluminum was built and measured.

The antenna configuration is depicted in Figure 2. The antenna model is defined by a long metallic cylinder with a $13.9 \mathrm{~mm}$ radius and $113.1 \mathrm{~mm}$ length, a short metallic cylinder with a $16.85 \mathrm{~mm}$ radius and $12.5 \mathrm{~mm}$ length, a circular ground plane with a $56.50 \mathrm{~mm}$ radius and the EBG structure, composed of two circular metal grids with the same radius size. An electric dipole located at $\lambda / 4$ from the bottom of the circular waveguide has been used to model the feeding of the waveguide in the simulations. The dipole is oriented in the $x$-axis parallel to the bottom of the waveguide.

Each grid is composed of a metallic sheet with twentyone square holes. Figure 3 shows the top view of the circular grids. The distance between the ground plane and the first metallic grid was established to prevent cavity resonances at the operating frequency whereas the distance between the two metallic grids was set to obtain the classical EBG mode that permits an efficient antenna performance.

The first step in the design process was choosing the unit cell shape of the periodic structure. Some candidates were studied, such as rectangles, triangles, circles, rhombs, and crosses. After an exhaustive study, the square hole was chosen because of its simplicity and its good response. The next 


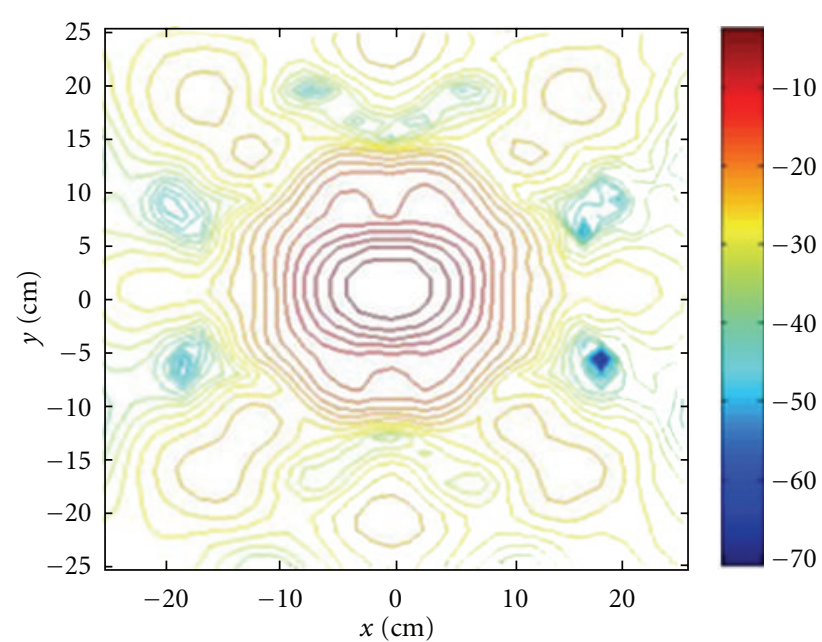

(a)

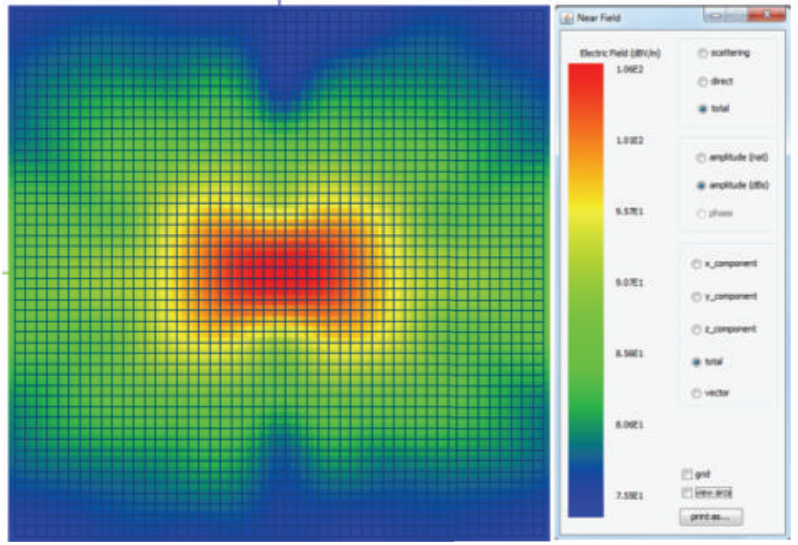

(b)

Figure 13: Near field results measured (a) and computed by the tool (b).

point was the optimization of the structure dimensions at a central frequency of $9 \mathrm{GHz}$. Several geometrical parameters (the distance between adjacent holes, the size of the holes, the number of holes, etc.) were optimized. After the analysis of the position of the periodic structures, the final conclusion is that the upper grid must be located $17.5 \mathrm{~mm}$ from the ground plane and the lower grid must be situated $3.5 \mathrm{~mm}$ from the ground plane. Those are the optimum physical dimensions to achieve the desirable radiation characteristics of high gain and low cross-polarization level. The remaining geometrical parameters related to the waveguide were fixed.

Regarding the resonance distances between the grids, the height of the lower grid from the ground plane was optimized within the range from $3 \mathrm{~mm}$ to $7.5 \mathrm{~mm}$ and the height of the upper grid from the ground plane was optimized within the range from 9 to $18 \mathrm{~mm}$. The optimization algorithm utilized to obtain the best values for both parameters was gradient descend and the optimization mask was defined to obtain low cross-polar level and high gain, according to the optimization goals in Table 1.

\section{Results}

The antenna prototype was measured in an anechoic chamber. Figure 4 depicts a photograph of the built antenna. The antenna was made of aluminum and weighed nearly $400 \mathrm{~g}$. The pair of metallic grids was kept fixed by four plastic screws. The screws crossed the ground plane and kept the three different parts together. The antenna was fed through a coaxial-rectangular transition plus a rectangular to circular waveguide transition to radiate linear polarization. Figure 5 shows the antenna located in the anechoic chamber.

Figures 6 and 7 show comparisons between the measurements and the simulated values for the two main radiation cuts at $9 \mathrm{GHz}$.

The EBG antenna provides the maximum radiation in the axial direction. Good agreement between real measure-
TABLE 1

\begin{tabular}{lcc}
\hline Theta range & $\begin{array}{r}\text { Level of the copolar } \\
\text { component }\end{array}$ & $\begin{array}{r}\text { Level of the cross-polar } \\
\text { component }\end{array}$ \\
\hline $0^{\circ}<\theta<10^{\circ}$ & $>-5 \mathrm{~dB}$ & $>40 \mathrm{~dB}$ \\
$10^{\circ}<\theta<20^{\circ}$ & $<-5 \mathrm{~dB}$ & $>40 \mathrm{~dB}$ \\
$20^{\circ}<\theta<30^{\circ}$ & $<-10 \mathrm{~dB}$ & $>40 \mathrm{~dB}$ \\
$30^{\circ}<\theta<90^{\circ}$ & $<-15 \mathrm{~dB}$ & $>40 \mathrm{~dB}$ \\
\hline
\end{tabular}

ments and computations was shown. The slight discrepancies between measurements and simulation values may be due to unwanted effects as well as nonidealities introduced by the four screws and the manufacturing errors. Moreover, the cross-polarization level in the $\mathrm{E}$ and $\mathrm{H}$ plane cuts is below $-40 \mathrm{~dB}$.

The maximum level of the gain is $15.6 \mathrm{dBi}$, given at theta $=0^{\circ}$ and phi $=0^{\circ}$. Figures 8 and 9 show the comparison between measurements and simulations of the gain for the $\mathrm{E}$ and $\mathrm{H}$ planes at $9 \mathrm{GHz}$.

Besides providing 2D Cartesian plots, the electromagnetic solver is able to display the $3 \mathrm{D}$ radiation pattern, as well as the current distribution, as it can be observed in Figures 10 and 11 .

Regarding the near field results, simulations and measurements have been conducted to compare the antenna behavior in a plane located $12 \mathrm{~cm}$ over the top of the antenna. A grid of $50 \times 50$ observation points has been established to compute and measure the near field values. Note that the distance between two consecutive points is $1 \mathrm{~cm}$. Therefore, the limits of the exploration area are $-25 \mathrm{~cm}$ and $25 \mathrm{~cm}$ in both axes, $x$ and $y$. Figure 12 shows the spatial configuration and the results provided by the computer tool. On the other hand, Figure 13 depicts the contour lines of the near field values measured in the anechoic chamber (on the left), and the simulated values (on the right). Units are in dBs. 


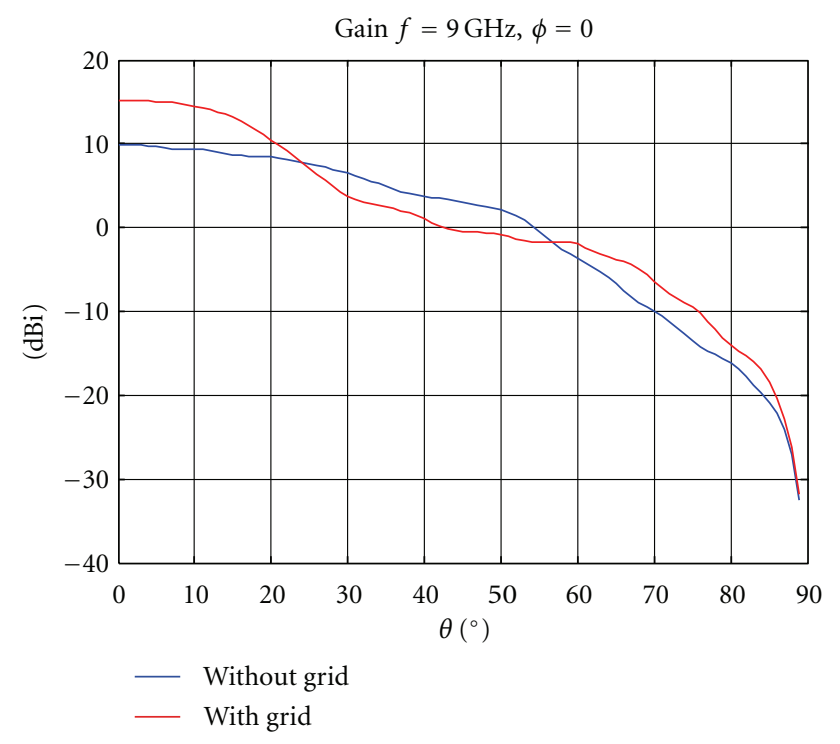

FIGURE 14: Comparison for the antenna gain in the E-plane cut with and without grids.

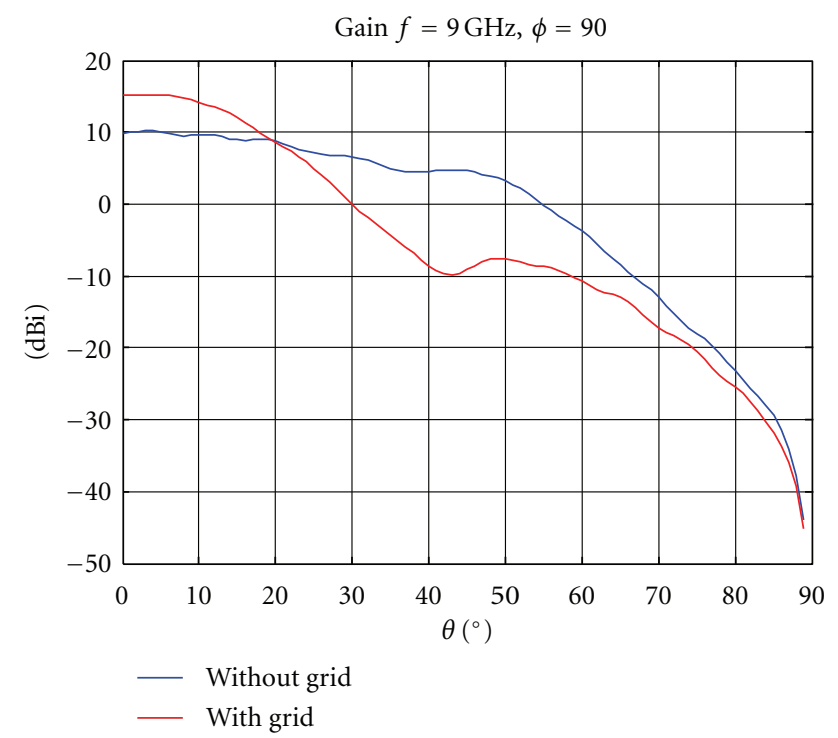

Figure 15: Comparison for the antenna gain in the H-plane cut with and without grids.

To verify the behavior of the EBG structure, several simulations were made to compare the results when the height of the cases were studied fixing the upper grid position at $17.5 \mathrm{~mm}$ and changing the lower grid position at h1 = $4.3 \mathrm{~mm}, \mathrm{~h} 2=5.1 \mathrm{~mm}, \mathrm{~h} 3=5.9 \mathrm{~mm}, \mathrm{~h} 4=6.7 \mathrm{~mm}$, and h5 $=7.5 \mathrm{~mm}$. A decrease in gain was proven when the height of the lower grid increases, in other words, when both grids are closer.

The comparison between the gain of the simple waveguide and the gain adding the EBG structure is shown in Figures 14 and 15 for the two main cuts. This comparison ensures that the effect of including the grids is a relevant enhancement in gain.

\section{Conclusions}

The design, optimization, and analysis of a compact EBG antenna operating at $9 \mathrm{GHz}$ have been presented in this paper. A powerful computer tool has been used to carry out those three processes. The antenna has been built and measured in order to validate the predictions provided by the electromagnetic solver. We have demonstrated that the effect of including the EBG structure is an enhancement in gain. The antenna is a good candidate for several applications due to its relatively high gain, excellent polarization purity, and compact size.

\section{Acknowledgment}

This paper has been supported, in part, by the Comunidad de Madrid Project S-2009/TIC1485 and by the Castilla-La Mancha Project PPII10-0192-0083, by the Spanish Department of Science, Technology Projects TEC 2010-15706 and CONSOLIDER-INGENIO No. CSD-2008-0068.

\section{References}

[1] M. Thèvenot, C. Cheype, A. Reineix, and B. Jecko, "Directive photonic-bandgap antennas," IEEE Transactions on Microwave Theory and Techniques, vol. 47, no. 11, pp. 2115-2122, 1999.

[2] G. K. Palikaras, A. P. Feresidis, and J. C. Vardaxoglou, "Cylindrical electromagnetic bandgap structures for directive base station antennas," IEEE Antennas and Wireless Propagation Letters, vol. 3, no. 1, pp. 87-89, 2004.

[3] A. R. Weily, K. P. Esselle, T. S. Bird, and B. C. Sanders, "Dual resonator 1-D EBG antenna with slot array feed for improved radiation bandwidth," IET Microwaves, Antennas and Propagation, vol. 1, no. 1, pp. 198-203, 2007.

[4] J.-W. Baik, S.-M. Han, C. Jeong, J. Jeong, and Y. S. Kim, "Compact ultra-wideband bandpass filter with EBG structure," IEEE Microwave and Wireless Components Letters, vol. 18, no. 10, Article ID 4639557, pp. 671-673, 2008.

[5] Y. Rahmat-Samii, "The Marvels of Electromagnetic Band Gap (EBG) Structures," Applied Computational Electromagnetics Society Journal, vol. 18, no. 3, pp. 1-10, 2003.

[6] G. V. Trentini, "Partially reflecting sheet arrays," IRE Transactions on Antennas and Propagation, vol. 4, pp. 666-671, 1956.

[7] C. Armenean, E. Perrin, M. Armenean, O. Beuf, F. Pilleul, and H. Saint-Jalmes, "RF induced temperature elevation near metallic wires in clinical magnetic resonance imaging," in Proceedings of the 25th Annual International Conference of the IEEE Engineering in Medicine and Biology Society, pp. 501-504, September 2003.

[8] I. González, E. García, F. S. De Adana, and M. F. Cátedra, "Monurbs: a parallelized fast multipole multilevel code for analyzing complex bodies modeled by NURBS surfaces," Applied Computational Electromagnetics Society Journal, vol. 23, no. 2, pp. 134-142, 2008.

[9] R. F. Harrington, Field Computation by Moment Methods, Macmillan, New York, NY, USA, 1968.

[10] W. C. Chew, J. Jin, E. Michielssen, and J. Song, Eds., Fast and Efficient Algorithms in Computational Electromagnetics, Artech-House, 2001.

[11] U. Jakobus and F. M. Landstorfer, "Improved PO-MM hybrid formulation for scattering from three-dimensional perfectly 
conducting bodies of arbitrary shape," IEEE Transactions on Antennas and Propagation, vol. 43, no. 2, pp. 162-169, 1995.

[12] C. Delgado, F. Cátedra, I. González, J. Gómez, and A. Tayebi, "Numerical approach for the fast analysis of radiation patterns of antennas in complex environments," in Proceedings of the Final Program and Book of Abstracts - International Workshop on Antenna Technology: Small Antennas, Innovative Structures and Materials (iWAT'10), pp. 1-4, 2010.

[13] http://www.mcs.anl.gov/research/projects/mpi/.

[14] P. M. Pardalos and M. G. C. Resende, Handbook of Applied Optimization, Oxford University Press, 2002.

[15] E.K. P. Chong and S. H. Zak, An Introduction to Optimization, John Wiley \& Sons, NJ, USA, 2008. 

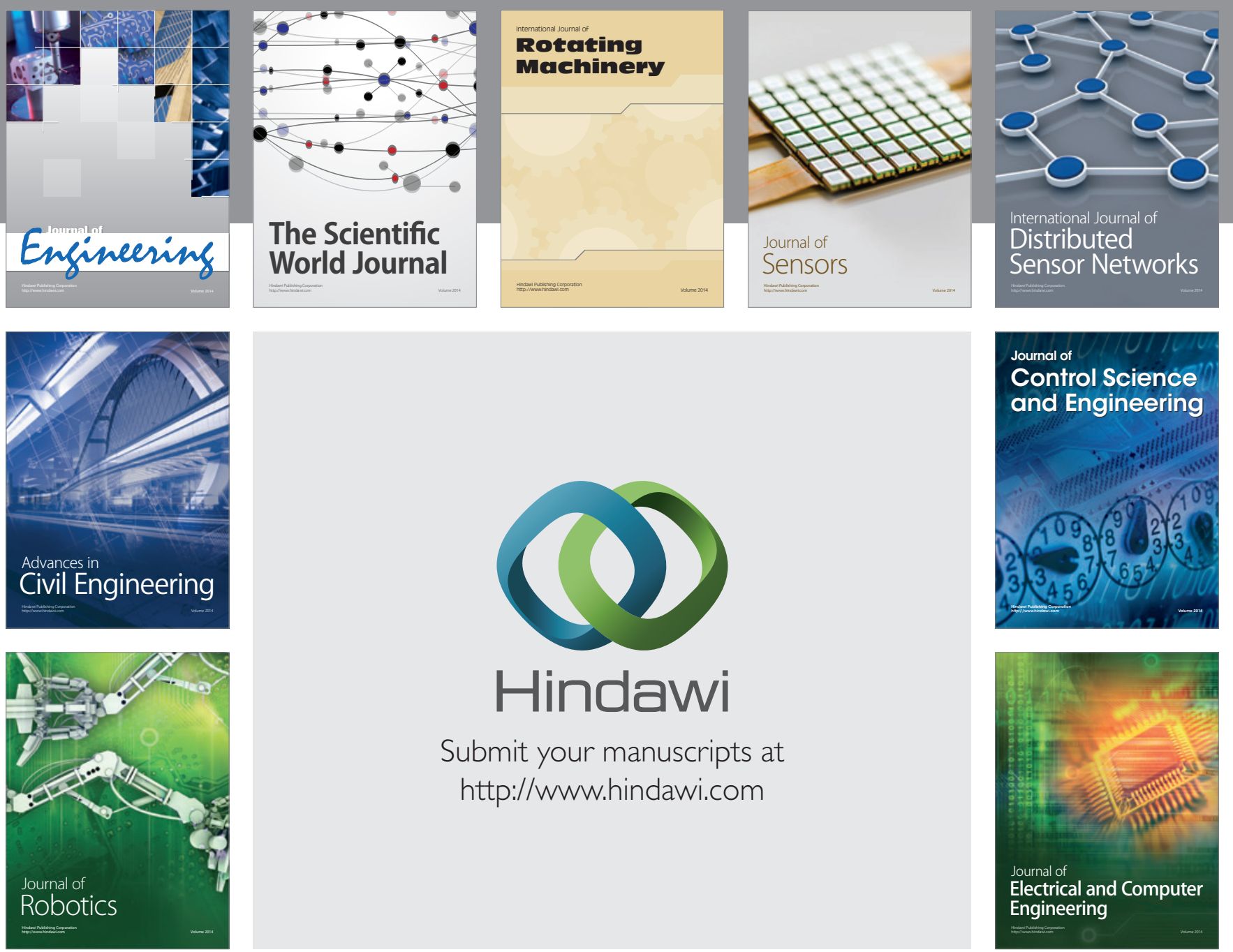

Submit your manuscripts at

http://www.hindawi.com
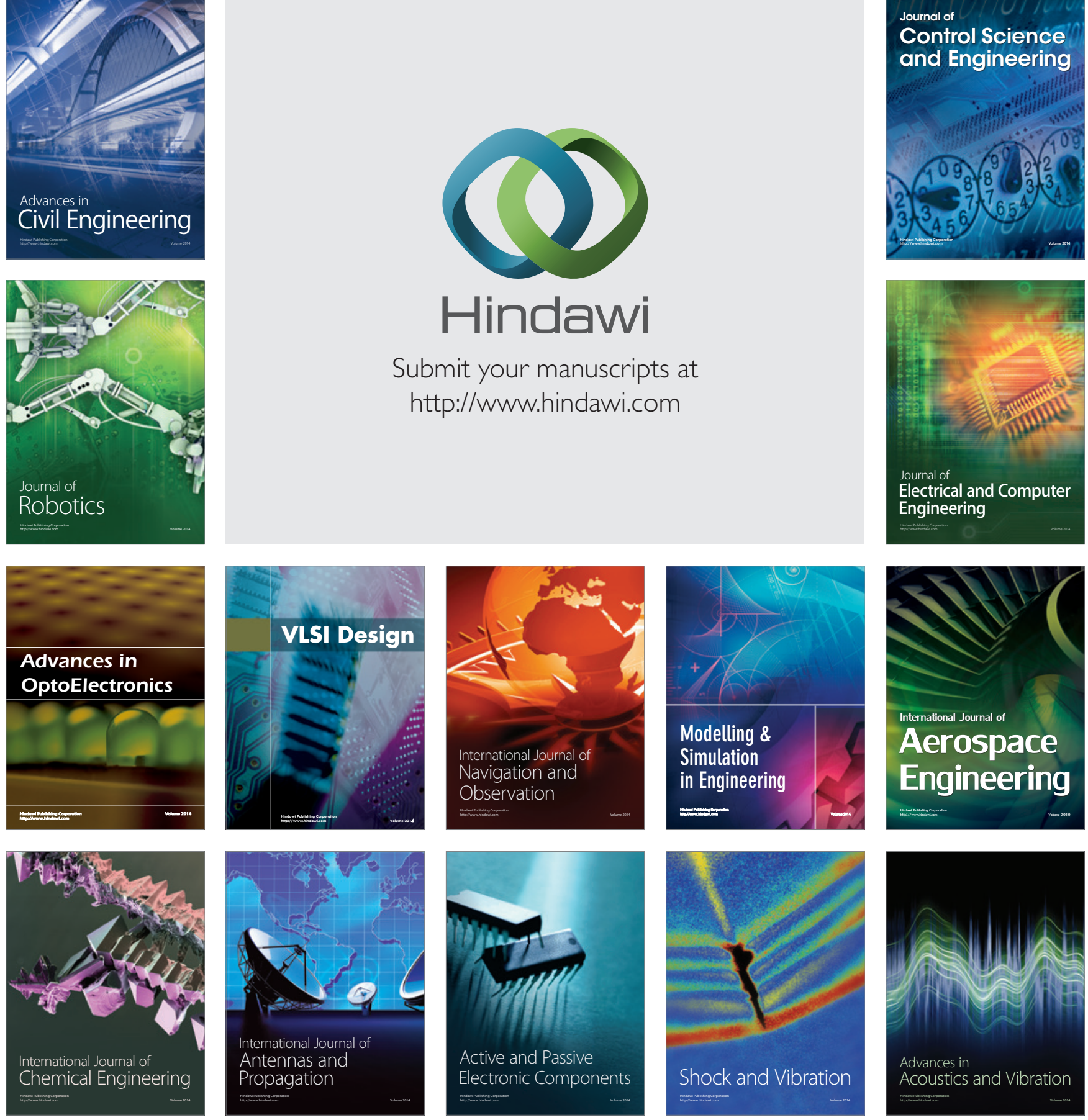